\title{
Efeito do treinamento funcional em variáveis antropométricas e no desempenho físico de mulheres treinadas
}

\section{Effect of functional training in anthropometrics variable and physical performance of trained women}

\author{
Pamela Alleman ${ }^{1}$ \\ Francine Caetano de Andrade Nogueira ${ }^{2}$ \\ Eberton Alves de Souza ${ }^{1}$ \\ Victor Hugo de Freitas ${ }^{1, *}$
}

\begin{abstract}
Resumo
Objetivo: O objetivo deste estudo foi analisar o efeito do treinamento funcional em varáveis antropométricas e no desempenho físico de mulheres treinadas. Métodos: Oito mulheres treinadas e saudáveis, participaram de sessões de treinamento funcional, 3 vezes por semana, por 7 semanas. As coletas de dados foram realizadas no primeiro dia de treinamento da primeira e da oitava semanas. Os dados coletados foram: massa corporal, estatura corporal, dobra cutâneas, flexibilidade, resistência muscular de abdome, salto vertical com contramovimento, agilidade, tempo de corrida por $2 \mathrm{~km}$ e percepção subjetiva de esforço (PSE) na corrida de $2 \mathrm{~km}$. A carga de treinamento foi monitorada pelo método da PSE da sessão. Resultados: Os principais resultados encontrados foram redução do percentual de gordura e melhorar o desempenho nos testes de resistência abdominal, de agilidade e de salto vertical $(P<0,05)$. Observa-se também uma provável redução na PSE na corrida de $2 \mathrm{~km}(3 / 11 / 87)$. Conclusão: Conclui-se que o treinamento funcional se mostrou efetivo para reduzir o percentual de gordura, para melhorar a resistência abdominal, a agilidade e o salto vertical, e que provavelmente reduziu PSE na corrida de $2 \mathrm{~km}$ de mulheres treinadas.
\end{abstract}

Palavras-chave: educação física e treinamento, atividade motora, saúde.

\begin{abstract}
Objective: The aim of this study was to analyze the effects of functional training on anthropometric variables and physical performance of trained healthy women. Methods: Eight trained and healthy women performed functional training sessions, 3 times a week, for 7 weeks. Data were collected on the first training day of the first and eighth weeks. The data collected were: body mass, body height, skin fold, flexibility, muscular resistance of the abdomen, countermovement vertical jump, agility, $2 \mathrm{~km}$ running time trial, and rating of perceived exertion (RPE) in a $2 \mathrm{~km}$ running time trial. Training load was monitored by the session RPE method. Results: The main results were that functional training reduced body fat and improved performance in abdominal resistance, agility, and vertical jump tests $(P<0.05)$. There was a probable reduction in the RPE in the $2 \mathrm{~km}$ running time trial (11/3/87). Conclusion: It was concluded that functional training was effective in reducing body fat, improving abdominal resistance, agility and vertical jump, and that it probably reduced RPE in the $2 \mathrm{~km}$ running time trial of trained women.
\end{abstract}

Keywords: Physical Education and training, motor activity, health.
Afiliação dos autores

${ }^{1}$ Curso de Educação Física, Centro Universitário Integrado, Campo Mourão, Paraná, Brasil.

${ }^{2}$ Faculdade de Psicologia, Universidade Federal de Juiz de Fora, Juiz de Fora, Minas Gerais, Brasil.

*Autor correspondente

Rodovia BR 158, Km 207, CEP 87300-970, Campo Mourão, Paraná. e-mail:

victor.freitas@grupointegrado.br

Conflito de interesses

Os autores declararam não haver conflito de interesses.

Processo de arbitragem

Recebido: 09/07/2018 Aprovado: 05/09/2018 


\section{Introdução}

O treinamento funcional é uma modalidade de exercício físico baseada na funcionalidade, ou seja, os exercícios executados durante a prática são estruturados com o objetivo de aperfeiçoar os movimento e as atividades realizadas na rotina diária de determinado público, sejam estes atletas ou não atletas $^{1,2}$. De acordo com a ideia de funcionalidade, grande parte dos estudos encontrados são focados em demonstrar o efeito do treinamento funcional na mobilidade, nas atividades da vida diárias e na qualidade de vida, principalmente em idosos ${ }^{3-6}$. No entanto, com a sua popularização, a ideia desta modalidade de treinamento tem sido utilizada não apenas para melhorar a funcionalidade de indivíduos praticantes, mas também como uma alternativa de treinamento físico para melhoria da saúde e qualidade de vida, e aperfeiçoamento das capacidades físicas e do fitness ${ }^{1,7}$

Há um consenso na literatura sobre a relação entre aptidão física e saúde ${ }^{8,9}$. Existem também inúmeras evidências apontando relação entre o sobrepeso e obesidade com o aparecimento de doenças como diabetes, hipertensão e doenças coronarianas ${ }^{10,11}$. Desse modo, a prática de exercício físico é aconselhada para o aumento da aptidão física, para a manutenção e redução da gordura corporal, e consequentemente para adquirir uma boa saúde ${ }^{9}$. De acordo com as recomendações do American Collegeof Sports Medicine (ACSM), um programa regular de exercícios para a manutenção das capacidades físicas e da boa saúde deve conter $\geq 150$ min/semana de exercícios cardiorrespiratórios em intensidade moderada, e 2-3 dias/semana de exercícios contra resistidos envolvendo grandes grupamentos musculares 9 . Desse modo, especula-se que utilizar um programa de treinamento físico, envolvendo exercícios com diferentes características, com intensidade moderada a intensa, popularmente chamado de treinamento funcional, com duração $\geq 150 \mathrm{~min} / \mathrm{semana}$ possa proporcionar manutenção ou melhora dos valores de gordura corporal e da aptidão física de sujeitos saudáveis.

Apesar do aumento da procura do treinamento funcional em centros voltados para a prática de exercício físico, são escassos os estudos que demonstram o efeito deste tipo de treinamento nas capacidades físicas como resistência, força, velocidade, agilidade e 0 fitness de mulheres saudáveis treinadas. Tomljanović et al. ${ }^{1}$, por exemplo, demonstraram a melhora no controle postural e coordenação de jovens fisicamente ativos submetidos a 5 semanas ( 3 vezes por semana) de treinamento funcional. No entanto, esses mesmos autores não encontraram alterações em variáveis antropométricas e no desempenho nos testes de agilidade shuttle-rune de salto vertical com contramovimento ${ }^{1}$. Sendo assim, evidências científicas podem reduzir o empirismo da prescrição e dos efeitos deste tipo treinamento. Baseado nesta lacuna, o objetivo deste estudo é analisar o efeito do treinamento funcional em varáveis antropométricas e no desempenho em testes físicos de mulheres saudáveis treinadas.

\section{Métodos}

\section{Sujeitos}

Participaram de forma voluntária deste estudo 8 mulheres (características na tabela 1), aparentemente saudáveis, praticantes de aulas coletivas de treinamento funcional por no mínimo cinco e no máximo quinze meses. Todas as mulheres praticavam as aulas com a mesma instrutora, com duração de 60 minutos, na mesma sala de uma academia de ginástica de uma cidade do interior do Paraná (aproximadamente 100 mil habitantes), com piso de grama sintética, às 9 horas da manhã, 3 vezes por semana. Foram adotados como critérios de inclusão: ter acima de 18 anos, frequentar as aulas regularmente, estar presente no dia do pré-teste e ser voluntário a participar do estudo. Os critérios de exclusão foram: se ausentar no momento pós teste, se ausentar por mais que 4 vezes, possuir limitação de movimentos por doença óssea, articular ou inflamatória, histórico de fraturas ou cirurgias recentes, possuir hipertensão, diabetes ou doença cardiovascular. Sendo assim, de acordo com os critérios de exclusão, dos 13 alunos iniciais, 5 foram excluídos.
O treinamento funcional foi realizado por um período de 7 semanas, com 3 sessões de treinos cada, totalizando 20 sessões. A duração de cada sessão foi de 50 minutos, e durante esse período, a carga de treinamento foi monitorada por meio do método da Percepção Subjetiva de Esforço da sessão (PSEsessão) 12. Os treinos foram realizados em forma de circuito, contendo de 8 a 15 exercícios, os quais foram realizados por 3 vezes (3 séries de 8 a 15 exercícios). Cada exercício foi realizado com duração de um minuto na primeira série, e 30 segundos na segunda e terceira séries, com intervalo de 15 segundos de um exercício para o outro e de 2 minutos entre as séries. Os exercícios realizados nas sessões de treino estão no quadro 1. Vale ressaltar que os pesquisadores não interferiram no treinamento realizado, e este estudo visou descrever a realidade prática. As coletas de dados foram realizadas no primeiro dia de treinamento da primeira e da oitava semanas, após 48 horas sem realizar exercícios. Os dados coletados foram: massa corporal, estatura corporal, dobra cutâneas, flexibilidade e resistência muscular de abdome, salto vertical com contramovimento, agilidade e tempo de corrida por $2 \mathrm{~km}$.

Este estudo foi aprovado por um Comitê de Ética em Pesquisa com seres humanos (parecer № 1.693.427), seguindo a Resolução 196/96 do Conselho Nacional de Saúde. Todos os procedimentos do estudo foram esclarecidos para os indivíduos, que assinaram um Termo de Consentimento Livre e Esclarecido expressando sua livre participação.

\section{Percepção subjetiva de esforço da sessão}

Para monitorar a carga interna de treinamento foi utilizado o método PSE-sessão 12. 10 minutos após cada sessão de treino, os alunos foram questionados sobre sua percepção de intensidade da sessão de treino como um todo e a resposta foi apontada em uma escala de percepção subjetiva de esforço (PSE) de 0 a 10 pontos 13 . A carga interna de treinamento diária foi obtida por meio do valor escolhido na escala de 0 a 10 , multiplicado pela duração da sessão de treino, em minutos, sendo expressa em unidades arbitrárias (U.A).

\section{Variáveis antropométricas}

Para mensurar o peso dos atletas, foi utilizada uma balança mecânica antropométrica (Welmy, W200a, Brasil) e a estatura foi mensurada por meio do estadiômetro anexado a balança (Welmy, W200a, Brasil). A partir dos valores de peso e altura foi calculado o índice de massa corporal dos sujeitos.

O tamanho das dobras cutâneas foram mensuradas por meio de um plicômetro científico (Sanny, Classic Sanny, Brasil). As dobras mensuradas foram, tricipital, subescapular, axilar media, supra ilíaca, peitoral, abdominal, coxa medial, com a densidade corporal sendo mensurado por meio do protocolo Jackson et al. ${ }^{14}$ de 7 dobras. O percentual de gordura foi calculado por meio da equação de $\mathrm{Siri}^{15}$.

\section{Flexibilidade}

Para avaliar a flexibilidade, foi realizado o teste de sentar e alcançar, utilizando o Banco de Wells. Neste teste, os sujeitos sentaram com as pernas unidas, joelhos estendidos, e com os pés apoiados na borda do banco de Wells. Estes deveria alcançar o mais distante possível ao longo da régua do banco com as duas mãos, uma sobre a outra, não sendo permitido flexionar os joelhos. Após três tentativas o avaliador anotava a maior distância alcançada em centímetro ${ }^{16}$.

\section{Resistência muscular abdominal}

Para avaliar a resistência muscular da região de abdômen, os sujeitos deveriam realizar o máximo de flexões de tronco possíveis em 1 minuto 16. Desta forma, eles iniciaram o teste em decúbito dorsal sobre um colchonete, com os joelhos flexionados, pés apoiados no chão e fixados pelo avaliador e braços cruzados em frente ao peito. Foram solicitadas flexões completas de tronco, ou seja, que tocassem os cotovelos nos joelhos.

\section{Procedimentos}




\section{Potência de membros inferiors}

Para inferir a potência de membros inferiores, foi realizado o teste de salto vertical com contramovimento. Os sujeitos iniciaram o teste na posição ortostática, sobre um tapete de contato (Cefise $\AA$; Nova Odessa, SP, Brazil), realizaram um agachamento de aproximadamente $90^{\circ}$ na articulação do joelho e um salto com ciclo alongamento-encurtamento. As mãos permaneceram por todo o teste apoiadas na cintura. Após 3 tentativas, com intervalo de 1 minuto entre elas, foi considerado o maior valor alcançado no teste, mensurada por meio do programa Jump System(Cefise ${ }^{\circledR}$; Nova Odessa, SP, Brazil).

\section{Agilidade}

Para avaliar a agilidade, foi utilizado o teste do Quadrado 17. Neste teste, um quadrado de $4 \mathrm{~m}^{2}$ é desenhado no chão, demarcados por cones de $50 \mathrm{~cm}$ em cada canto. Conforme o protocolo, cada participante iniciou o teste na posição ortostática, $30 \mathrm{~cm}$ atrás do cone de partida, correu em direção ao cone posicionado na diagonal, seguindo para o cone a sua direita, para o cone na diagonal, e finalmente, retornou para o cone de partida. Era necessário tocar os cones com uma das mãos. Uma fotocélula (Speed Test Fit, Cefise $\AA^{\circ}$; Nova Odessa, Brazil) foi posicionada no cone de partida para mensurar a duração do teste. Foram realizadas 3 tentativas e considerada a melhor entre elas.

\section{Tempo de corrida por $2 \mathrm{~km}$}

Neste teste, cada participante deveria percorrer sem interrupção uma distância de $2 \mathrm{~km}$ em uma esteira (Moviment, LX160, Brasil) com o menor tempo possível. Ao final de cada minuto, foi questionado sobre sua PSE naquele momento, devendo apontar sua resposta na escala de percepção subjetiva de esforço de 0 a 10 pontos 13 . O tempo de duração na corrida por $2 \mathrm{~km}$ e a média da PSE coletada durante a corrida foram registrados.

\section{Análise estatística}

Para testar o efeito do treinamento funcional em varáveis antropométricas e no desempenho físico de mulheres saudáveis, foi utilizado o teste-t para variáveis dependentes. O software utilizado para realizar as análises foi o Statistica (v.8.0, StatSoft $\circledast$, Tulsa, Ok), considerando o valor de $P<0,05$. Para verificar a chance das mudanças nas variáveis analisadas como efeito do treinamento funcional serem maiores ou menores que a mínima mudança detectável $(\mathrm{MMD}=0.2 \mathrm{X}$ desvio padrão no momento pré), foi utilizado o método de inferência baseada em magnitude ${ }^{18}$. As chances quantitativas de encontrar diferenças foram interpretadas qualitativamente como: $<1 \%$, quase certamente não; $1-5 \%$, muito improvável; 5-25\%, improvável, 25 $75 \%$, possível; $75-95 \%$, provável; $>95 \%-99 \%$, muito provável; $>99 \%$, quase certo. Se as chances de encontrar mudanças forem $>5 \%$ para ambos os lados positivo e negativo $(>5 / X X />5)$, os dados foram interpretados como obscuros.

\section{Resultados}

A PSE e a PSE-sessão das 20 sessões de treinamento foram descritas nas figuras 1 e 2. Nota-se, neste período, uma oscilação diária entre as cargas. Percebe-se também que as médias das PSEs estão entre 3 (moderado) e 6 (entre difícil e muito difícil). Apenas na última sessão de treinamento o valor de PSE apresentado foi 2.

Os resultados obtidos nas avaliações realizadas nos momentos pré e pós treinamento estão na tabela 1. Nota-se que, após as 7 semanas de treinamento funcional, as mulheres avaliadas apresentaram menor percentual de gordura $(P=0,03)$, apesar do mesmo peso e IMC. Além disso, elas apresentaram melhor desempenho nos testes de SVC $(P=0,04)$ e de agilidade $(P=0,04)$ no momento pós treinamento comparado com o momento pré. Nenhuma outra variável foi diferente quando os momentos pré e pós foram comparados $(P>0,05)$.

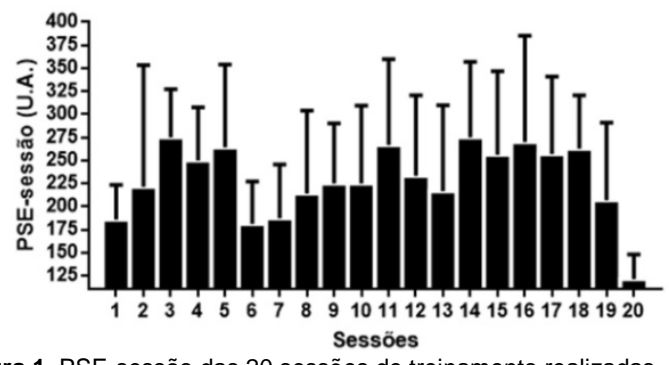

Figura 1. PSE-sessão das 20 sessões de treinamento realizadas.

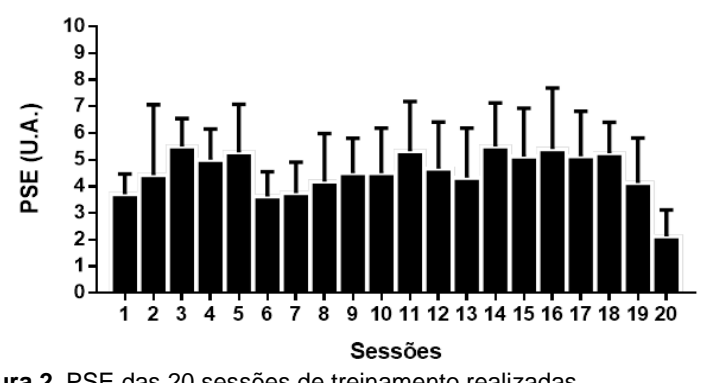

Figura 2. PSE das 20 sessões de treinamento realizadas.

\section{Tabela 1}

Variáveis antropométricas e de desempenho nos momentos pré e póstreinamento $(n=8)$.

\begin{tabular}{|c|c|c|c|c|}
\hline $\begin{array}{l}\text { Idade } \\
\text { Estatura }\end{array}$ & $\begin{array}{c}28,78 \pm 8,20 \\
1,65 \pm 0,07\end{array}$ & & & \\
\hline & Pré & Pós & $\begin{array}{l}\text { Valor } \\
\text { de } P\end{array}$ & Diferença da MMD \\
\hline $\begin{array}{l}\text { Peso } \\
\text { IMC }\end{array}$ & $\begin{array}{l}62,38 \pm 7,51 \\
23,47 \pm 2,35\end{array}$ & $\begin{array}{l}61,77 \pm 7,39 \\
23,25 \pm 2,35\end{array}$ & $\begin{array}{l}0,08 \\
0,09\end{array}$ & $\begin{array}{c}\text { Maior/trivial/menor } \\
0 / 99 / 1 \\
0 / 97 / 3\end{array}$ \\
\hline $\begin{array}{l}\text { Porcentual } \\
\text { de gordura }\end{array}$ & $33,24 \pm 4,76$ & $32,08 \pm 5,60$ & 0,03 & $0 / 32 / 68$ \\
\hline Flexibilidade & $29,25 \pm 8,07$ & $30,00 \pm 7,62$ & 0,39 & $16 / 83 / 1$ \\
\hline $\begin{array}{l}\text { Resistência } \\
\text { Abdominal }\end{array}$ & $31,88 \pm 5,38$ & $33,63 \pm 3,38$ & 0,16 & $72 / 26 / 2$ \\
\hline $\begin{array}{l}\text { Agilidade } \\
\mathrm{CMJ}(\mathrm{cm})\end{array}$ & $\begin{array}{c}6,94 \pm 0,33 \\
22,13 \pm 3,21\end{array}$ & $\begin{array}{c}6,68 \pm 0,41 \\
23,70 \pm 3,97\end{array}$ & $\begin{array}{l}0,04 \\
0,04\end{array}$ & $\begin{array}{l}1 / 4 / 95 \\
90 / 9 / 1\end{array}$ \\
\hline $\begin{array}{l}\text { Tempo de } \\
\text { corrida por } \\
2 \mathrm{~km} \text { (min) }\end{array}$ & $14,25 \pm 1,58$ & $14,12 \pm 1,50$ & 0,59 & $5 / 72 / 23$ \\
\hline PSE $-2 \mathrm{~km}$ & $4,39 \pm 0,85$ & $3,85 \pm 1,06$ & 0,12 & $3 / 11 / 87$ \\
\hline
\end{tabular}

As chances das diferenças nas variáveis serem diferentes da MMD estão apresentadas na tabela 1. Os resultados indicaram que é possível que o percentual de gordura das mulheres diminua com o treinamento, com o peso e altura apresentando resultado trivial. É improvável que a flexibilidade e o tempo de corrida tenha mudado com o treinamento. No entanto, é possível que a resistência abdominal tenha aumentado e que a PSE no teste de corrida tenha diminuído, e é provável que 0 desempenho nos testes de agilidade e CMJ tenha melhorado.

\section{Discussão}

Os principais resultados encontrados foram que o treinamento funcional se mostrou efetivo para reduzir 0 percentual de gordura e melhorar o desempenho em testes de resistência abdominal, de agilidade e de salto vertical em mulheres treinadas. Os resultados mostraram que o treinamento funcional não modificou a flexibilidade nem o tempo de corrida destas mulheres, apesar da provável redução apresentada na PSE durante o teste de corrida de $2 \mathrm{~km}$.

Ao contrário do que foi reportado por Tomljanović et al. ${ }^{1}$, o percentual de gordura das mulheres treinadas que participaram do presente estudo diminuiu após apenas 7 semanas de treinamento funcional. O treinamento físico, principalmente quando realizado em alta intensidade, pode reduzir o percentual de gordura, aumentar o metabolismo de lipídios, mesmo quando não se observa alterações no peso corporal ${ }^{19}$. Tal alteração na composição corporal está relacionado a redução de marcadores relacionados ao risco cardiovascular ${ }^{20}$. Neves et al..$^{21}$, reportaram que 8 semanas de treinamento funcional realizado em forma de circuito reduziu a gordura corporal total de mulheres na pós- 
menopausa. No entanto, vale ressaltar que no estudo de Neves et al. $^{21}$, as mulheres eram destreinadas, o que garante a originalidade do presente estudo.

A melhoria do desempenho nos testes de resistência abdominal, de agilidade e de salto vertical observada no presente estudo também são diferentes dos encontrados por Tomljanović et al. ${ }^{1}$, que demonstraram que o treinamento funcional é mais eficiente para melhoria do controle postural e da coordenação. A possível explicação para os resultados encontrados no presente estudo pode estar relacionada aos exercícios executados com carga e velocidade para membros inferiores, aos exercícios pliométricos, e para o fortalecimento da região abdominal prescritos durante as sessões de treino. Treinamento de potência e pliométrico mostram-se efetivos para melhorar o desempenho em testes de agilidade e de salto vertical em atletas ${ }^{22-24}$. Além disso, 8 semanas de treinamento realizado em forma de circuito aumentou a resistência abdominal de mulheres obesas sedentárias ${ }^{25}$. Sendo assim, os resultados encontrados mostram que o treinamento funcional, quando programados com exercícios executados com carga e velocidade, pliométricos e para o fortalecimento da região abdominal proporcionam efeito positivo nos testes de resistência abdominal, de agilidade e de salto vertical.

$\mathrm{O}$ treinamento funcional, no entanto, não proporcionou efeito no tempo de corrida em esteira por $2 \mathrm{~km}$. A redução do tempo na corrida era esperado uma vez que uma diminuição no tempo de $5 \mathrm{~km}$ de corrida foi reportado em atletas de resistência submetidos a um período de treinamento de força explosiva ${ }^{26}$. No entanto, vale ressaltar o efeito observado na PSE durante a corrida de $2 \mathrm{~km}$, sugerindo um possível efeito positivo do treinamento funcional no desempenho deste teste. De acordo com o modelo psicobiológico, a PSE é a responsável por regular a intensidade do exercício de resistência ${ }^{27}$. Sendo assim, é possível que os sujeitos tenham se dedicado mais ao teste de corrida de $2 \mathrm{~km}$ no momento pré do que no momento pós, o que ocasionou a não diferença estatística no tempo de corrida.

Nenhum efeito do treinamento funcional foi observado no teste de flexibilidade. Uma possível explicação para este resultado encontrado pode ser o pouco volume de treinamento para aperfeiçoar essa capacidade física. Resultados similares foram observados por dos Reis Filho et $\mathrm{al}^{25}$, após 8 semanas de treinamento em circuito com mulheres obesas sedentárias. De acordo com o ACSM, o treinamento adequado para melhorar a flexibilidade de adultos saudáveis deve conter exercícios de flexibilidade, repetidos 2-4 vezes para cada cadeia muscular, com duração de 10-30 segundos cada exercício, realizados mais que 2 vezes na semana ${ }^{28}$. Deste modo, aparentemente, o treinamento realizado no presente estudo foi insuficiente para melhorar a flexibilidade de mulheres treinadas.

\section{Conclusão}

Conclui-se que o treinamento funcional realizado 3 vezes por semana, por um período de 7 semanas, quando programado com exercícios de potência, pliométricos e para o fortalecimento da região abdominal proporciona efeito positivo nos testes de resistência abdominal, de agilidade e de salto vertical, além de diminuir o percentual de gordura de mulheres saudáveis treinadas. É provável também que, este tipo de treinamento, reduza a PSE durante a corrida de $2 \mathrm{~km}$.

Estes resultados são importante a medida em que ajudam a reduzir o empirismo da prescrição e dos efeitos deste tipo treinamento, que atualmente é amplamente difundido em centros de atividades físicas e praticado por vários grupos de pessoas.

Neste sentido, pesquisas futuras são necessárias a fim de verificar outros tipos de periodização do treinamento funcional para comparação dos resultados. Da mesma maneira, sugeremse estudos posteriores que avaliem a melhoria destas variáveis em mulheres destreinadas, em idosos, em gestantes e em obesos para um aprofundamento dos benefícios do treinamento funcional.

\section{Referências}

1. Tomljanović M, Spasić M, Gabrilo G, Uljević O, Foretić N. Effects of five weeks of functional vs. traditional resistance training on anthropometric and motor performance variables Kinesiology. 2011;43(2):145-54.

2. Da Silva-Grigoletto ME, Brito CJ, Heredia JR. Functional training: functional for what and for whom? Rev Bras Cineantropom Desempenho Hum. 2014;16(6):714-9.

3. Liu C, Shiroy DM, Jones LY, Clark DO. Systematic review of functional training on muscle strength, physical functioning, and activities of daily living in older adults. European review of aging and physical activity official journal of the European Group for Research into Elderly and Physical Activity. 2014;11:95-106.

4. Whitehurst MA, Johnson BL, Parker CM, Brown LE, Ford AM. The benefits of a functional exercise circuit for older adults. J Strength Cond Res. 2005;19(3):647-51

5. de Vreede PL, Samson MM, van Meeteren NL, Duursma SA, Verhaar HJ. Functional-task exercise versus resistance strength exercise to improve daily function in older women: a randomized, controlled trial. J Am Geriatr Soc. 2005;53(1):2-10.

6. Gine-Garriga M, Guerra M, Pages E, Manini TM, Jimenez R, Unnithan VB. The effect of functional circuit training on physical frailty in frail older adults: a randomized controlled trial. J Aging Phys Act. 2010;18(4):401-24.

7. Weiss T, Kreitinger J, Wilde H, Wiora C, Steege M, Dalleck L, et al. Effect of Functional Resistance Training on Muscular Fitness Outcomes in Young Adults. Journal of Exercise Science \& Fitness. 2010;8(2):113-22.

8. Blair SN, Kohl HW, 3rd, Barlow CE, Paffenbarger RS, Jr., Gibbons LW Macera CA. Changes in physical fitness and all-cause mortality. A prospective study of healthy and unhealthy men. JAMA. 1995;273(14):1093-8.

9. Garber CE, Blissmer B, Deschenes MR, Franklin BA, Lamonte MJ, Lee IM, et al. American College of Sports Medicine position stand. Quantity and quality of exercise for developing and maintaining cardiorespiratory, musculoskeletal, and neuromotor fitness in apparently healthy adults: guidance for prescribing exercise. Med Sci Sports Exerc. 2011;43(7):133459.

10. Mokdad AH, Ford ES, Bowman BA, Dietz WH, Vinicor F, Bales VS, et al. Prevalence of obesity, diabetes, and obesity-related health risk factors, 2001. JAMA. 2003;289(1):76-9.

11. Must A, Spadano J, Coakley EH, Field AE, Colditz G, Dietz WH. The disease burden associated with overweight and obesity. JAMA 1999;282(16):1523-9.

12. Foster C, Florhaug JA, Franklin J, Gottschall L, Hrovatin LA, Parker S, et al. A new approach to monitoring exercise training. J Strength Cond Res. 2001;15(1):109-15

13. Borg G, Hassmen $P$, Lagerstrom M. Perceived exertion related to hear rate and blood lactate during arm and leg exercise. Eur J Appl Physio Occup Physiol. 1987;56(6):679-85.

14. Jackson AS, Pollock ML, Ward A. Generalized equations for predicting body density of women. Med Sci Sports Exerc. 1980;12(3):175-81.

15. Siri WE. Body composition from fluids spaces and density: analyses of methods. In: Techniques for measuring body composition. Washington: National Academy of Science and Natural Resource Council; 1961.

16. Mathwes DK. Medidas e Avaliação em Educação Física. Rio de Janeiro: Guanabara; 1986.

17. de Freitas VH, Ramos SP, Bara-Filho MG, Freitas DG, Coimbra DR Cecchini $R$, et al. Effect of cold water immersion performed on successive days on physical performance, muscle damage, and inflammatory,
hormonal, and oxidative stress markers in volleyball players. J Strength hormonal, and oxid.
Cond Res. 2017.

18. Batterham AM, Hopkins WG. Making meaningful inferences about magnitudes. Int J Sports Physiol Perform. 2006;1(1):50-7.

19. Tremblay A, Simoneau JA, Bouchard C. Impact of exercise intensity on body fatness and skeletal muscle metabolism. Metabolism. 1994;43(7):814-8.

20. Arciero PJ, Gentile CL, Martin-Pressman R, Ormsbee MJ, Everett M Zwicky L, et al. Increased dietary protein and combined high intensity aerobic and resistance exercise improves body fat distribution and cardiovascular risk factors. Int J Sport Nutr Exerc Metab. 2006;16(4):37392.

21. Neves LM, Fortaleza AC, Rossi FE, Diniz TA, de Castro MR, de Aro BL, et al. Effect of a short-term functional training program on body composition in postmenopausal women. Rev Bras Ginecol Obstet. 2014;36(9):404-9.

22. Miller MG, Herniman JJ, Ricard MD, Cheatham CC, Michael TJ. The effects of a 6-week plyometric training program on agility. J Sports Sci Med. 2006;5(3):459-65.

23. Vaczi M, Tollar J, Meszler B, Juhasz I, Karsai I. Short-term high intensity plyometric training program improves strength, power and agility in male soccer players. J Hum Kinet. 2013:36:17-26.

24. de Freitas VH, Nakamura FY, de Andrade FC, Pereira LA, Coimbra DR, Bara-Filho M. Pre-competitive physical training and markers of performance, stress and recovery in young volleyball athletes. Rev Bras Cineantropom Desempenho Hum. 2015;17(1):31-40.

25. dos Reis Filho AD, Silva MLS, Fett CA, Lima WP. Efeitos do treinamento em circuito ou caminhada após oito semanas de intervenção na composição corporal e aptidão física de mulheres obesas sedentárias.Revista Brasileira de Obesidade, Nutrição e Emagrecimento. 2008;2(1):498-507.

26. Paavolainen L, Hakkinen K, Hamalainen I, Nummela A, Rusko H Explosive-strength training improves $5-\mathrm{km}$ running time by improving running economy and muscle power. J Appl Physiol (1985) 1999;86(5):1527-33

27. Smirmaul BPC, Dantas JL, Nakamura FY, Pereira G. The psychobiological model: a new explanation to intensity regulation and (in)tolerance in endurance exercise. Bras Educ Fís Esporte. 2013;27(2):333-40.

28. American College of Sports Medicine Position Stand. The recommended quantity and quality of exercise for developing and maintaining cardiorespiratory and muscular fitness, and flexibility in healthy adults. Med Sci Sports Exerc. 1998;30(6):975-9 